ARTIGO

ORIGINAL

\title{
A redução dos níveis de cortisol sanguíneo através da técnica de relaxamento progressivo em nadadores
}

\author{
Maurício Gattás Bara Filhoㄹ, Luiz Carlos Scipião Ribeiro², \\ Renato Miranda ${ }^{3}$ e Mônica Tavares Teixeira ${ }^{4}$
}

\section{RESUMO}

Objetivo: Analisar os efeitos da técnica de relaxamento progressivo na redução/controle dos níveis de cortisol sanguíneo em nadadores durante determinado período de treinamentos. Metodologia: Nadadores de ambos os sexos (n $=23$ ) foram divididos em dois grupos: experimental $-\mathrm{GE}$ $(\mathrm{n}=11)$, submetido a tratamento com a técnica de relaxamento progressivo duas vezes por semana; de controle $\mathrm{GC}(\mathrm{n}=12)$, submetido a tratamento placebo nas mesmas condições. Os tratamentos foram realizados durante sete semanas, com três coletas de sangue (pré, mid e pós-testes). Resultados: A partir da não-normalidade da distribuição indicada pelo teste de Kolmogorov-Smirnov (Dmax $=3,61>$ Dcrítico $=0,276, \mathrm{NC}=95 \%$ ), decidiu-se pela análise exploratória dos dados, que revelou os seguintes valores da mediana do nível de cortisol sanguíneo $(\mathrm{ng} / \mathrm{ml})$ para os grupos nos pré, mid e pós-testes, respectivamente: $\mathrm{GE}=$ 140,7; 95,5 e 86,5, e GC $=141,1 ; 132,8$ e 138,6. Além de observar maior redução da mediana no GE, os valores deste grupo apresentaram-se mais próximos da mediana no pós-teste, o que não ocorreu com o GC, que permaneceu inalterado em relação ao pré-teste. Para corroborar essa tendência, utilizou-se o teste de Mann-Whitney e observou-

1. Professor Assistente da Faculdade de Educação Física e Desportos da Universidade Federal de Juiz de Fora. Mestre em Educação Física pela Universidade Gama Filho - RJ.

2. Sub-Reitor Acadêmico da Universidade Estácio de Sá - RJ. Doutor em Psicofisiologia - Boston University.

3. Professor Adjunto da Faculdade de Educação Física e Desportos da Universidade Federal de Juiz de Fora - MG. Doutor em Educação Física pela Universidade Gama Filho - RJ.

4. Graduanda em Medicina pela Universidade Federal de Juiz de Fora MG.

Recebido em 9/8/01

2ํㅗ versão recebida em 23/11/01

Aceito em 30/5/02

Endereço para correspondência:

Maurício Gattás Bara Filho

Rua São Sebastião, 1.295/901

36015-410 - Juiz de Fora, MG

E-mail: mgbara@terra.com.br se uma diferença significativa $(\mathrm{p}<0,004)$ na soma de postos para GE, ao contrário do GC ( $\mathrm{p}>0,05)$, o que demonstra maior redução dos níveis de cortisol sanguíneo no GE em relação ao GC. Conclusões: Os resultados indicam que há influência do tratamento experimental (relaxamento progressivo) na redução dos níveis de cortisol sanguíneo. Isso demonstra intensa relação psicofisiológica entre os processos do organismo humano, indicando a necessidade da utilização de estratégias de controle do treinamento desportivo para evitar o estresse excessivo.

Palavras-chave: Cortisol. Psicofisiologia. Treinamento desportivo. Relaxamento.

\section{ABSTRACT}

Reduction of blood cortisol levels through the progressive relaxation technique in swimmers

Purpose: To analyze the effects of the progressive relaxation technique to reduce/control blood cortisol levels in swimmers during a period of training. Methods: Swimmers of both sexes $(n=23)$ were divided into two groups: experimental $-E G(n=11)$, which was submitted to the progressive relaxation technique session twice a week; control $C G(n=12)$, which was submitted to a placebo treatment under the same conditions. The treatments lasted for seven weeks with three blood samples (pre, mid and post tests). Results: Starting from the distribution non-normality showed by the Kolmogorov-Smirnov test (D) $\max =3.61$ > Dcritical $=0.276, \mathrm{p}=0.05)$, the exploratory analysis reveals the following cortisol $(\mathrm{ng} / \mathrm{ml})$ median values in the pre, mid and post tests, respectively: $E G=140.7 ; 95.5$, and 86.5, and $C G=141.1 ; 132.8$, and 138.6. Besides this greater reduction in the EG median values, the EG values were closer to median in the posttest, which did not happenned in the CG. To corroborate this tendency, the MannWhitney test was used, depicting a significant statistical difference ( $p<0.004)$ for $E G$, which did not occur in $C G$ ( $p$ $>0.05$ ), depicting a greater cortisol level reduction in EG compared to the CG. Conclusion: The results indicate that the experimental treatment (progressive relaxation) influenced the reduction of blood cortisol levels. These results 
show an intense psychophysiologic relationship between human body processes, indicating the need to use training strategies to prevent excessive stress.

Key words: Cortisol. Psychophysiology. Physical training. Relaxation.

\section{INTRODUÇÃO}

O esporte competitivo é um fator causador de estresse em conseqüência de variáveis fisiológicas, biomecânicas, psicológicas e metabólicas que exercem papel fundamental no desempenho esportivo. Intensidades e volumes adequados de treinamento físico aumentam a capacidade fisiológica do indivíduo, normalmente levando-o a melhor performance. No entanto, o treinamento desportivo com cargas excessivas é considerado um estressor negativo para o organismo, podendo causar estresse desconfortável, perigoso e prejudicial à performance e à saúde do atleta ${ }^{1-3}$.

$\mathrm{O}$ fenômeno do estresse é definido por McGrath (apud Weinberg e Gould, p. 96) ${ }^{4}$ como "um desequilíbrio substancial entre a demanda (física e/ou psíquica) e a capacidade de resposta, sob condições nas quais o fracasso para suprir a demanda tem importantes conseqüências".

Qualquer tipo de estímulo estressor (físico e/ou psicológico) pode desencadear reações psicofisiológicas que acabam resultando em hiperfunção do sistema nervoso simpático e do sistema endócrino, mais particularmente, da glândula supra-renal. Perante tal desequilíbrio, torna-se função do hipotálamo e do sistema nervoso parassimpático auxiliar na adaptação ou recuperação do organismo e na manutenção de condições homeostáticas ${ }^{5,6}$.

Assim, aumento nos níveis de estresse ativa o sistema endócrino, resultando na maior liberação de hormônios glicocorticóides, tal como o cortisol, pela glândula suprarenal. Maior liberação de cortisol sugere refletir situações de perda de controle, depressão e, principalmente, distresse (estresse negativo). Ao contrário, níveis mais baixos, porém normais de cortisol, indicam maior autocontrole, previsibilidade de ação e envolvimento prazeroso e motivante na tarefa $\mathrm{a}^{5,7,8}$.

O cortisol tem sido bastante utilizado como medida fisiológica em estudos sobre estresse no esporte relacionado com a carga de treinamentos ${ }^{2,5,9-11}$ ou em outras áreas ${ }^{7,8}$.

$\mathrm{O}$ estado de relaxamento causa no indivíduo mudanças psicofisiológicas que são opostas ou revertem o efeito do sistema nervoso simpático (responsável pelas reações de ativação do corpo humano). $\mathrm{O}$ atleta, para atingir sua eficiência atlética, deve utilizar-se de técnicas de relaxamento, pois elas objetivam a otimização do descanso, bem como a redução e o controle da ansiedade e do estresse, permi- tindo, assim, a organização de todas as forças e dirigindoas para a performance $e^{12-14}$.

O presente estudo objetivou analisar os efeitos da técnica de relaxamento progressivo na redução/controle dos níveis de cortisol sanguíneo em nadadores durante determinado período de treinamentos.

\section{MATERIAL E MÉTODOS}

O presente estudo caracteriza-se como uma pesquisa experimental verdadeira por apresentar controle rigoroso e manipulação direta da variável experimental. Além disso, tem-se a presença de um grupo de controle e randomização das amostras ${ }^{15}$.

Todos os atletas participaram de uma competição de controle ao final da segunda semana de tratamento e de uma competição alvo uma semana após o pós-teste. Dessa maneira, acredita-se que em nenhum dos testes houve influência do processo competitivo no nível de cortisol sanguíneo devido ao fato de ambos os grupos estarem sendo submetidos ao mesmo processo de planificação do treinamento.

\section{Amostra}

Vinte e três nadadores, entre 14 e 20 anos, de ambos os sexos, que treinavam e competiam por um clube da cidade de Juiz de Fora-MG na época da coleta, foram divididos randomicamente em grupo experimental $(\mathrm{n}=11)$ e grupo de controle $(n=12)$, após um emparelhamento por sexo e anos de treinamento.

Todos os atletas e seus respectivos responsáveis assinaram um termo de consentimento explicando todos os procedimentos do estudo, atestando a participação voluntária e concordando com a utilização dos dados para publicação científica.

\section{Delineamento do estudo}

O estudo desenvolveu-se durante sete semanas, com um total de três coletas de dados: no início da primeira semana (pré-teste), e no final da quarta (mid-teste) e sétima (pósteste) semanas.

O grupo experimental recebeu o tratamento através da técnica de relaxamento progressivo durante as sete semanas com duas sessões semanais de 20 a 30 minutos, como proposto por Becker Jr. e Samulski ${ }^{13}$ e Suinn ${ }^{16}$.

Utilizou-se no presente estudo a técnica de relaxamento progressivo idealizada por Sigmund Jacobson na década de 30 e que se tornou a base de várias técnicas de relaxamento modernas e uma das mais aplicadas e conhecidas no mundo para regulação do estresse. O nome "progressivo" corresponde à progressão do relaxamento de um músculo para outro até que todos os grandes grupos musculares estejam relaxados ${ }^{13,16}$. 
O grande diferencial desse método decorre do fato de ele ser ativo (envolve contração e relaxamento), quando comparado com os outros, e de possuir orientação mais fisiológica do que psicológica. Dessa maneira, sugere-se que os atletas possam considerá-lo mais atrativo, de assimilação mais fácil e rápida, otimizando o estado de relaxamento psicofisiológico nos indivíduos ${ }^{13,17}$.

O tratamento placebo foi realizado nas mesmas condições do experimental, com duas reuniões semanais, nas quais os atletas eram conscientes de estar realizando um treinamento psicofisiológico como o grupo experimental. Diversas atividades foram realizadas utilizando informações cognitivas (discussões sobre o estresse do treinamento desportivo, problemas com colegas, técnicos e cobrança dos pais), técnicas motivacionais (determinação de objetivos para competições futuras) e visualização motora de técnicas de nado e tática de prova.

No entanto, essas técnicas, apesar de ser itens da psicologia do esporte, não influenciam na redução do estresse psicofisiológico do treinamento desportivo, visto que cada técnica foi aplicada em uma ou no máximo duas sessões do tratamento, impossibilitando, assim, um ganho de determinada habilidade pelo treinamento.

Gould e Udry ${ }^{17}$ e Vealey ${ }^{18}$ justificam o fato de o grupo de controle receber tratamento placebo através de técnicas motivacionais, evitando o problema de esse grupo não ter contato com o pesquisador e, conseqüentemente, possíveis desvios nos resultados.

Todo o programa de atividades foi realizado dentro do ambiente de treinamentos dos atletas, o que, para Gould e Udry ${ }^{17}$ e Vealey ${ }^{18}$, reflete melhor as condições de estresse dos atletas em relação aos testes realizados em laboratórios. Essa característica classifica o estudo como ecologicamente válido.

Como nenhum dos grupos sabia se se integrava ao experimental ou ao de controle, cabendo apenas aos pesquisadores esse conhecimento, a pesquisa pode ser caracterizada como simples-cega ${ }^{19}$.

\section{Coleta de dados}

As três coletas de dados foram realizadas no horário principal de treinamento dos atletas (entre 4 e 6 PM). Os atletas foram orientados a permanecer em total repouso por 15 minutos antes da coleta de sangue para a análise do cortisol. O sangue coletado era imediatamente transportado para o laboratório responsável pelas análises, que eram realizadas através do teste VIDAS cortisol, que permite medir quantitativamente e de forma direta o nível de cortisol presente no soro ou plasma humano. Os valores considerados normais do cortisol sérico no referido teste encontram-se entre 40 e $150 \mathrm{ng} / \mathrm{ml}$ durante o período da tarde.

\section{RESULTADOS}

Os dados dos grupos em relação às variáveis sexo, idade e anos de treinamento estão apresentados na tabela 1, na qual se observa homogeneidade dessas variáveis entre os grupos experimental e de controle.

Decidiu-se por testar formalmente a hipótese de normalidade da distribuição. Procedeu-se ao teste de Kolmogorov-Smirnov, obtendo-se o valor de Dmax $=3,61$ superior ao Dcrítico $=0,276$ para o nível de significância de $95 \%$. Esses resultados rejeitam a hipótese de normalidade, indicando a distribuição como não-normal, sugerindo a utilização de uma estatística não paramétrica na análise dos dados.

O fato de a distribuição ser não-normal indicou a necessidade de realizar uma análise exploratória dos dados, calculando mediana e diferenças interquartil, e não a estatística descritiva com média, desvio padrão e coeficiente de variação.

Os resultados obtidos na análise exploratória estão apresentados na tabela 2.

Observa-se, na tabela 2 , redução dos valores das medianas entre o pré e o pós-teste no grupo experimental, ao contrário do grupo de controle, no qual a mediana se manteve com valores muito próximos.

$\mathrm{O}$ grupo de controle manteve-se praticamente inalterado entre o pré e o pós-teste e o grupo experimental evolui tanto na redução da mediana como na aproximação dos valores em torno da mediana do pré para o pós-teste. Esses resultados indicam uma influência positiva do tratamento experimental na redução dos níveis de cortisol sanguíneo nos nadadores.

Constatou-se através do teste de Mann-Whitney que a diferença entre a soma dos postos é estatisticamente significativa ( $p<0,004)$ para GE, o que não ocorre com GC, indicando, portanto, a eficácia do tratamento experimental (relaxamento progressivo) na redução dos níveis de cortisol sanguíneo.

\begin{tabular}{|c|c|c|c|}
\hline \multicolumn{4}{|c|}{$\begin{array}{c}\text { TABELA } 1 \\
\text { Distribuição dos atletas segundo as variáveis } \\
\text { sexo, idade e tempo de treinamento }\end{array}$} \\
\hline Grupo & $\begin{array}{l}\text { Sexo (n) } \\
\text { M - masculino } \\
\text { F - Feminino }\end{array}$ & $\begin{array}{l}\text { Idade } \\
\text { (média } \\
\text { - anos) }\end{array}$ & $\begin{array}{c}\text { Tempo de } \\
\text { treinamento } \\
\text { (média - anos) }\end{array}$ \\
\hline Experimental & $\begin{array}{l}M-7 \\
F-4\end{array}$ & $17,1 \pm 2,5$ & $6,9 \pm 2,3$ \\
\hline Controle & $\begin{array}{l}M-9 \\
F-3\end{array}$ & $16,2 \pm 2,1$ & $7,2 \pm 2,6$ \\
\hline
\end{tabular}


TABELA 2

Níveis de cortisol (ng/ml) no grupo experimental (GE) e grupo de controle (GC)

\begin{tabular}{lccccccc}
\hline & \multicolumn{3}{c}{ Controle } & & \multicolumn{3}{c}{ Experimental* } \\
\cline { 2 - 3 } \cline { 7 - 8 } & Pré-teste & Mid-teste & Pós-teste & & Pré-teste & Mid-teste & Pós-teste \\
Mediana & 141,1 & 132,8 & 138,6 & & 140,7 & 95,5 & 86,5 \\
Q1 & 103,2 & 125,3 & 92,9 & & 82,4 & 80,1 & 82,5 \\
Q3 & 181,8 & 175,1 & 176,6 & & 155,9 & 134,7 & 122,9 \\
Amplitude & 78,6 & 49,8 & 83,7 & & 73,5 & 54,6 & 40,4 \\
interquartil & & & & & & \\
\hline
\end{tabular}

* $\mathrm{P}<0,004 ; \mathrm{Q} 1$ - primeiro quartil; $\mathrm{Q} 2$ - segundo quartil.

Esses resultados corroboram os estudos de Toivanen ${ }^{7} \mathrm{e}$ Toivanen et al. ${ }^{8}$, que observaram aumento do índice de cortisol no grupo de controle e diminuição no grupo experimental, porém, em uma população de não-atletas. Nesses estudos, também foi utilizada uma técnica de relaxamento como forma de intervenção.

\section{DISCUSSÃO}

Os resultados apontam alguns aspectos originais do presente estudo. O mais relevante encontra-se no fato de o organismo humano ser estudado dentro de uma simultaneidade psicofisiológica, o que foi demonstrando no controle de variáveis fisiológicas através de uma técnica mencionada pela psicologia do esporte. Estudos previamente publicados abordam os efeitos fisiológicos das cargas de treinamento ${ }^{2,9-11,21-23,}$ nos quais, entretanto, não se observa a indicação de estratégias alternativas de controle dessas variáveis fisiológicas como indicado no presente estudo com a técnica de relaxamento.

Os estudos de Toivanen ${ }^{7}$ e Toivanen et al. ${ }^{8}$ são semelhantes ao presente; no entanto, pesquisam uma população de trabalhadoras, o que difere do presente estudo, que pesquisou uma população de atletas, caracterizando outro aspecto original.

A implementação de técnicas de relaxamento no processo de treinamento desportivo pode otimizar a redução dos níveis de estresse. A técnica de relaxamento progressivo mostrou-se um método eficaz na redução dos níveis de cortisol sanguíneo, uma das respostas fisiológicas negativas do sistema nervoso simpático provocada pelo estresse das cargas de treinamento.

Isso demonstra a intensa relação psicofisiológica presente entre os processos internos do organismo humano e a necessidade de os profissionais envolvidos no treinamento de atletas buscarem estratégias de preparação que venham a influenciar psicofisiologicamente o desenvolvimento dos atletas.
Dentro do planejamento de atividades, treinadores, médicos do esporte, psicólogos do esporte, fisiologistas e atletas devem compreender que a utilização de estratégias como técnicas de relaxamento durante uma a três horas semanais pode minimizar os possíveis efeitos negativos dos treinamentos, auxiliando na recuperação psicofisiológica e objetivando melhor aproveitamento em busca da melhoria da performance desportiva.

Estudos futuros devem considerar a utilização de um grupo amostral maior, bem como pesquisar a influência de técnicas psicológicas em diferentes variáveis, como creatina quinase, ácido lático e catecolaminas.

Todos os autores declararam não haver qualquer potencial conflito de interesses referente a este artigo.

\section{REFERÊNCIAS}

1. Goss JD. Hardness and mood disturbances in swimmers while overtraining. Journal of Sports and Exercise Psychology 1994;16:135-49.

2. Kirwan JP, Costill DL, Flynn MG, Mitchell JB, Fink WJ, Neufer PD, et al. Physiological responses to successive days of intense training in competitive swimmers. Med Sci Sports Exerc 1988;20:255-9.

3. Tierney J. Stress in age-group swimmers. Swimming Technique 1998; Feb-Apr:9-14.

4. Weinberg R, Gould D. Foundations of sport and exercise psychology. Champaign: Human Kinetics, 1995.

5. Brandão ML, Lachat JJ. Noções básicas de neuroanatomia. In: Brandão ML, organizador. Psicofisiologia. São Paulo: Atheneu, 1995:1-17.

6. Brandão ML. Comportamento emocional. In: Brandão ML, organizador. Psicofisiologia. São Paulo: Atheneu, 1995:105-26.

7. Toivanen H. Occupational stress in working women and the benefits of relaxation training [Tese de Doutorado]. University of Kuopio, Finland, 1994.

8. Toivanen H, Lansimies E, Jokela V, Helin P, Penttila I, Hanninen O. Plasma levels of adrenal hormones in working women during an economical recession and threat of unemployment: impact of regular relaxation training. Journal of Psychophysiology 1996;10:36-48. 
9. Flynn MG, Pizza FX, Boone Jr JB, Andres FF, Michaud TA, RodriguezZayas JR. Indices of training stress during competitive running and swimming seasons. Int J Sports Med 1994;15:21-6.

10. Flynn MG, Carroll KK, Hall HL, Bushman BA, Brolinson PG, Weideman CA. Cross training: indices of training stress and performance. Med Sci Sports Exerc 1998;30:294-300.

11. Hooper SL, Mackinnon LT, Howard A, Gordon RD, Bachmann AW. Markers for monitoring overtraining and recovery. Med Sci Sports Exerc 1995;27:106-12.

12. Becker Jr B. A preparação psicológica do atleta. Revista Brasileira de Educação Física e Desporto 1981;10:15-8.

13. Becker Jr B, Samulski D. Manual do treinamento psicológico para o esporte. Feevale, 1998.

14. Cox RH. Sport psychology: concepts and applications. Dubuque: Brown and Bench-Mark, 1994.

15. Isaac S, Michael WB. Handbook in research and evaluation. San Diego: Edits Publishers, 1982.
16. Suinn RM. Sete etapas para a performance de pico. São Paulo: Manole, 1998.

17. Gould D, Udry E. Psychological skills for enhancing performance: arousal regulation strategies. Med Sci Sports Exerc 1994;26:478-85.

18. Vealey RS. Current status and prominent issues in sport psychology interventions. Med Sci Sports Exerc 1994;26:495-502.

19. Stratton P, Hayes N. Dicionário de psicologia. São Paulo: Pioneira, 1994.

20. Sokal RR, Rohlf J. Biometry. New York: Freeman \& Co., 1969.

21. Derman W, Schwellnus MP, Lambert MI, Emms M, Sinclair-Smith C, Kirby C, et al. The 'worn-out athlete': a clinical approach to chronic fatigue in athletes. J Sports Sci 1997;15:341-51.

22. Lehmann MJ, Lormes W, Opitz-Gress A, Steinacker JM, Netzer N, Foster $\mathrm{C}$, et al. Training and overtraining: an overview and experimental results in endurance sports. J Sports Med Phys Fitness 1997;37:7-17.

23. Costill DL, Thomas R, Robergs RA, Pascoe D, Lambert C, Barr S, et al. Adaptations to swimming training: influence to training volume. Med Sci Sports Exerc 1991;23:371-7. 